\section{$4 \quad$ IDENTIFY NOVEL THERAPIES FOR VASCULAR DISEASE AND REGENERATIVE MEDICINE BASED ON THE LANDMARK DISCOVERY OF INDUCED PLURIPOTENT STEM CELLS}

R Sterritt, R Caines, A Margariti. The Wellcome-Wolfson Building, Centre for Experimental Medicine, Queen's University Belfast

\subsection{6/heartjnl-2018-SCF.14}

Cardiovascular disease (CVD) is the leading cause of death globally, in 2013 it was responsible for $30 \%$ of all deaths worldwide. It has been shown that endothelial dysfunction is a major pathological mechanism that precedes CVD, yet these damaged endothelial cells (ECs) display poor regenerative capabilities. Current therapy is inadequate or unsuitable for a significant number of patients, however it is thought that the ability to replace damaged ECs could serve as a valuable therapeutic option for the future. Recent capability to generate induced pluripotent stem cells (iPSCs) from adult cells and differentiate these towards ECs holds great promise in the area of regenerative medicine. Nevertheless, the differentiation process involved is complex and not fully understood. An appreciation of a variety of molecular mechanisms is required in order to enhance protocols that could be replicated for novel therapies. This project has derived ECs from iPSCs using a reliable protocol. Results here confirmed successful differentiation with an up-regulation in important endothelial markers at the mRNA level alongside positive immunofluorescent staining. These differentiated cells were characteristically similar to mature ECs. In order to expose underlying mechanisms of EC differentiation, the novel gene SETSIP was shown to be upregulated during this process. Further investigation to stimulate differentiating cells with a SETSIP peptide noted an induction in endothelial markers. Finally, experiments involving epigenetic drugs induced changes in both endothelial marker and SETSIP expression. This project has elucidated the role of SETSIP and data shown here may be important in advancing therapeutic potential through the development of more robust protocols concerning EC differentiation.

\section{RESIDENT S100 $3 / \mathrm{SCA}^{+}{ }^{+}$MULTIPOTENT VASCULAR STEM CELLS UNDERGO MYOGENIC AND VASCULOGENIC DIFFERENTIATION IN VITRO}

${ }^{1}$ Denise Burtenshaw, ${ }^{1}$ Emma Fitzpatrick, ${ }^{2}$ Weimin Liu ${ }^{2}$ David Morrow, ${ }^{2}$ Eileen M Redmond, ${ }^{1}$ Paul A Cahill. 'Vascular Biology and Therapeutics Laboratory, School of Biotechnology Faculty of Science and Health, Dublin City University, Dublin 9, Ireland; ' ${ }^{2}$ Department of Surgery, University of Rochester Medical Centre, Rochester, NY, USA

\subsection{6/heartjil-2018-SCF.15}

Arteriosclerosis is an important age-dependent disease that encompasses atherosclerosis, in-stent restenosis, autologous bypass grafting and transplant arteriosclerosis. Vascular smooth muscle (vSMC)-like accumulation is a key event leading to intimal-medial thickening (IMT), vessel remodelling and an important marker of subclinical disease. Vascular stem cell progeny in addition to de-differentiated SMC and/or SMC derived from endothelial-mesenchymal transition (EndoMT) are all reported to contribute to IMT as they become activated/dysfunctional, differentiate down vascular and myeloid lineages and subsequently dictate, in-part, vessel remodelling. In this study we examined the multipotent potential of a specialised population of rat adult resident multipotent vascular stem cells (rMVSC) located within the vessel wall and their capability to differentiate down both myogenic (muscle) and vasculogenic (endothelial) lineages when given the appropriate stimulus. Using Sca1-eGFP transgenic mice in vivo, there was a significant increase in the number of $\mathrm{Sca}^{+}$cells within the intima of the left carotid artery (LCA) following partial carotid artery ligation-induced injury after 3 days that co-localised with endothelial nitric oxide synthase (eNOS) and $\mathrm{CD} 31^{+}$positive cells, when compared to the sham-operated control vessels and the contralateral right carotid artery (RCA). The number of Sca1-eGFP ${ }^{+}$cells significantly increased over time within the adventitial, medial and neointimal layers following ligation-induced injury after 7 and 14 days, respectively. In vitro, S100 $\beta / \mathrm{Sca}^{+}$rat MVSCs cultured in vasculogenic inductive media for 7 days underwent differentiation to an endothelial cell phenotype characterised by the appearance of a cobblestone morphology and increased eNOS expression. In contrast, MVSC exposed to media supplemented with TGF- $\beta 1$ for 7 days underwent myogenic differentiation to SMC-like cell. These data suggest that resident S100 $\beta / \mathrm{Sca}^{+}$ MVSCs are capable of both myogenic and vasculogenic differentiation depending on the inductive stimulus and may contribute in part to intimal medial thickening (IMT) and endothelial regeneration following injury in vivo.

\section{PARALLEL ASSESSMENT OF CELL VIABILITY IN CARDIAC AND CANCER CELLS FOLLOWING TREATMENT WITH SUNITINIB}

Domenica McBride, Abdulfattah Alhazmi, Sarunya Laovitthayanggoon, Marie Boyd, Susan Currie. Strathclyde Institute of Pharmacy and Biomedical Sciences, University of Strathclyde, 161 Cathedral Street, Glasgow

\subsection{6/heartjnl-2018-SCF.16}

Various cardiopathological effects have been observed following chemotherapy treatment in cancer patients, due to anticancer drug-induced cardiotoxicity (CTX). A retrospective study of cancer survivors reported a $50 \%$ and $10 \%$ incidence of hypertension and heart failure respectively following treatment with the tyrosine kinase inhibitor sunitinib, licensed to treat pancreatic neuroendocrine tumours. The cellular mechanisms underlying CTX are not known. Here, for the first time, we compare the potency of sunitinib in both cardiac cells (primary cardiac fibroblasts (CFs)) and cancer cells (a pancreatic adenocarcinoma cell line (PANC-1)).

Adult rat CFs were isolated by bulk collagenase digestion, maintained in culture and used between passages 1-2. PANC1 cells, from previously-frozen stocks, were used between passages 41-49. Cells were treated with sunitinib $(0-10 \mu \mathrm{M}$ in CFs; $0-100 \mu \mathrm{M}$ in PANC-1) for 24 hour prior to epifluorescent imaging for phenotypic assessment. Cell viability was examined by alamar blue assays following 24 hour sunitinib treatment $(0-100 \mu \mathrm{M})$.

Overall, results indicated increased sensitivity of CFs to sunitinib compared with PANC-1 cells. Phenotypic changes indicative of cell death, including appearance of intracellular vacuoles, were evident in CFs following $1 \mu \mathrm{M}$ sunitinib treatment whereas similar effects were not induced until $10 \mu \mathrm{M}$ treatment in PANC-1 cells. Alamar blue assays demonstrated a dramatic increase in CF death compared to PANC-1 death following treatment with $10 \mu \mathrm{M}$ sunitinib (11.6 \pm 0.02 vs 56.5 